\title{
Data and Safety Monitoring Boards: Some Enduring Questions
}

\author{
Charles J. Kowalski and Jan L. Hewett
}

\section{Introduction}

Data Safety and Monitoring Boards (DSMBs) ${ }^{1}$ were introduced in the 1960s to monitor data in clinical trials to ensure subject safety. It was thought important that DSMB members be experts in the field(s) of interest, but not otherwise involved in the study (e.g., sponsors and investigators) in order to maximize objectivity. Since then, the use of DSMBs has increased dramatically, ${ }^{2}$ and their scope has expanded to include scientific issues - in particular, to avoid bias that can result when trials are stopped early because of evidence that one treatment has greater efficacy or causes greater harm than another; or that no treatment is likely to do better than any other.

Four principles guiding the operation of DSMBs were offered by Susan Ellenberg and her co-authors: ${ }^{3}$

Principle 1. The primary responsibilities of a DMC [DSMB] are to: (1) safeguard the interests of study patients; (2) preserve the integrity and credibility of the trial in order that future patients may be treated optimally; and (3) ensure that definitive and reliable results are available in a timely way to the medical community.

Principle 2. The DMC should have multidisciplinary representation, including physicians from relevant medical specialties and biostatisticians. In many

Charles J. Kowalski, Ph.D., is a Professor of Dentistry at the University of Michigan School of Dentistry in Ann Arbor, MI. He is also a statistician at the Center for Statistical Consultation and Research and a co-chair of the Health Sciences IRB. He earned his Ph.D. (1968) in biostatistics from the University of Michigan at Ann Arbor. Jan L. Hewett, J.D., is the Director of the University of Michigan Medical School Institutional Review Board (IRBMED) at the University of Michigan. She earned her B.S.N. from Georgetown University in Washington, D.C., and J.D. from the University of Michigan at Ann Arbor. cases, other experts such as bioethicists, epidemiologists, and basic scientists should also be included.

Principle 3. The DMC should have membership limited to individuals free of apparent significant conflicts of interest, whether they are financial, intellectual, professional, or regulatory in nature.

Principle 4. The DMC members should ideally be the only individuals to whom the data analysis center provides results on relative efficacy and safety of study treatments.

The first two principles are uncontroversial and widely accepted. Our discussion focuses on principles 3 and 4 . The third principle includes the proscription of investigators (those conducting the trial) from DSMB membership. The thought is that study integrity and credibility are compromised if individuals with apparent conflicts of interest are in a position to determine, e.g., whether to stop a trial. Justification for the fourth principle is to minimize the risk of prejudging outcomes based on unreliable results from limited data. The hope is to avoid declining accrual rates over time, inappropriate termination of the trial yielding equivocal results, and final results at odds with prematurely published interim results. ${ }^{4}$ As to affecting recruitment, Robert Wells and colleagues ${ }^{5}$ noted,

[I]t was felt that if the group that developed the study....knew about interim data as it accrued, they might question the validity of their initial impression that there were no important differences among the therapies under study [equipoise]. Patient accrual would then decrease because the clinicians involved would not offer the study to their patients. Instead they would pick the 'best' treatment as established by early data trends and 
thus interfere with the eventual demonstration of the superiority of one therapy over another.

There is limited evidence regarding how emerging data might influence recruitment, but Sarah Edwards and colleagues 6 found, "Willingness to undergo randomization drops as prospective participants are given more preliminary data and as they are made aware of any accumulating evidence of differential efficacy." This evidence can be either in terms of the magnitude of the difference or the corresponding p-value. As magnitudes increase or p-values fall, there is less willingness to participate. This shows that individuals will assimilate preliminary information and take it into account in their decision making.

The principles noted above have been widely accepted: DSMBs have been called a "growth industry,"7 and recent Food and Drug Administration (FDA) ${ }^{8}$ guidance extols the virtues of DSMBs. A full 34 pages are used to detail the structure and function of DSMBs and to emphasize the belief of the FDA that keeping interim results confidential removes serious threats to trial integrity. Citing the regulations, sponsors are to take appropriate measures to minimize bias, and this includes remaining ignorant of interim analyses of the data: "such knowledge can bias the outcome of the study by inappropriately influencing its continued conduct or the plan of analyses. Unblinded interim data and the results of comparative interim analyses, therefore, should generally not be accessible by anyone other than DMC members or the statistician(s) performing these analyses and presenting them to the DMC."

But even the most enthusiastic proponents do not claim that DSMBs are always needed, or that they be routinely employed. The following criteria were suggested in determining the need for a DSMB:

(1) Is the trial intended to provide definitive information about effectiveness and/or safety of a medical intervention?

(2) Are there prior data to suggest that the intervention being studied has the potential to induce potentially unacceptable toxicity?

(3) Is the trial evaluating mortality or another major endpoint, such that inferiority of one treatment arm has safety as well as effectiveness implications?

(4) Would it be ethically important for the trial to stop early if the primary question addressed has been definitively answered, even if secondary questions or complete safety information were not yet fully addressed?
A DSMB would likely be appropriate if two or more of the above criteria were met, and probably not warranted if none were met. ${ }^{9}$ Our stance, on the contrary, is that DSMBs carry enough ethical baggage that their use should be considered only when benefits clearly outweigh the disadvantages to be detailed below. We make the further observation that, in the rare instances in which DSMB use is warranted, consent forms clearly spell out its existence, its function, and exactly what information will - and will not - be shared with subjects as the trial evolves.

\section{DSMB Disadvantages}

Despite the popularity of DSMBs, questions have been raised about some of their functions, and it is the purpose of this paper to summarize the opposing literature, and to further develop objections based on U.S. regulations. We do not argue for a complete elimination of the DSMB, but feel that enough issues will have been raised to question the popularity or "growth of the industry." Again, as noted earlier, our discussion focuses on two issues regarding DSMBs: (1) excluding investigators from membership, and (2) keeping the accumulating data secret, i.e., not sharing interim results with participants, those considering participation, and the physicians responsible for the care of these individuals. We consider first the question of whether investigators should have a role in the monitoring process.

\section{Principled Principal Investigators}

We begin with a paper by Curtis Meinert ${ }^{10}$ that takes umbrage to the suggestion that investigators are especially vulnerable to unjustified enthusiasm (or pessimism) based on unreliable early trends in the data and need to be protected from themselves in this regard. Meinert put forth four conditions he considered necessary for adequate monitoring (timeliness, completeness, competency, and freedom), and argued that investigators "have the necessary training, skills, expertise, and information to enable them to perform in an informed and competent fashion." He pointed out that investigators would be the ones who would be blamed, regardless of who actually did the monitoring, should a trial be judged to have been too long or too short. He noted, "Investigators have a duty to halt or modify a trial if and when its ethical base, broadly referred to as clinical equipoise, has eroded. Implicit to the duty is the need to be informed of accumulating results of one's own trial and of other related trials." He thought further, "There is irony in that, in the case of trials where we experiment on human beings, those who do so cannot be 'trusted' to see the data they gen- 
erate while they so experiment," and concluded, "the best safeguards against harm to subjects of research reside in informed and caring investigators answering to demanding and vigilant IRBs [Institutional Review Boards]."

The problems encountered when investigators are not kept in the loop are further exacerbated when trial subjects are patients of the investigators. It is of special concern when those responsible for the care of the subjects in the trial are deprived of information that might help guide that care. Benjamin Freedman ${ }^{11}$ thought that physicians had an obligation to obtain any and all information that might impact the care of their patients:

Although [DSMBs] are useful in keeping the conduct of a trial free of bias, they cannot resolve the investigators' ethical difficulties. A clinician is not merely obligated to treat a patient on the basis of the information he or she currently has, but is also required to discover information that would be relevant to treatment decisions. If interim results would disturb equipoise, the investigators are obliged to gather and use that information. Their agreement to remain ignorant of preliminary results would, by definition, be an unethical agreement, just as a failure to call up the laboratory to find out a patient's test results is unethical.

Should a patient/subject discover that her physician had failed to make every effort to acquire information that might be useful to guide her treatment, this would surely erode the physician-patient trust relationship. Serious damage to the image of the physician as a practicing, empathetic professional who is primarily concerned with the patient as an individual could result. Spread of a skeptical view towards the medical research establishment would seriously undermine the entire research enterprise, especially research involving human subjects.

Samuel and Deborah Hellman agreed. ${ }^{12}$ They thought that physicians should eschew trials that attempted to restrict their access to data since "their ability to act in the patient's best interests will be limited." They continued,

$[\mathrm{I}] \mathrm{f}$ physicians continue the randomization when they have a definite opinion about the efficacy of the experimental drug, they are not acting in accordance with the requirements of the doctorpatient relationship. Furthermore, as their opinion becomes more firm, stopping the randomization may not be enough. Physicians may be ethically required to treat the patients formerly placed in the control group with the therapy that now seems probably effective.

They made these recommendations realizing the impact they would have on the study design. They looked to the development of other, less ethically problematic, experimental designs as an outcome clearly favored to abrogating the doctor-patient relationship. Indeed, they thought that "[p]hysicians can acquire knowledge through methods other than the randomized clinical trial. Such knowledge, acquired over time and less formally than that is required in a randomized clinical trial [RCT], may be of great value to a patient." We applaud this focus on the patient and the notion that knowledge acquired outside of RCTs may be of value to the patient (and that not all knowledge acquired via the RCT necessarily has value).

Richard Lilford and colleagues ${ }^{13}$ considered, "Withholding...information that patients might find useful is at best paternalistic, at worst authoritarian and arguably unnecessary." They thought that while, often, "ignorance is perpetuated to stop potential participants voting with their feet," there are times when releasing interim information might in fact increase recruitment. ${ }^{14}$ Finally, they thought it ironic that while recent guidelines admonished investigators to keep up-to-date with the literature and modify the information they give to patients accordingly, data from their own trial would nevertheless be kept secret. They concluded that since the meaning of interim data will vary from person to person, decisions made on the basis of such data should be individual decisions - not made for the collective behind closed doors.

The National Bioethics Advisory Commission (NBAC) recognized that "unless individual investigators understand their ethical responsibilities no regulatory system will function properly" and quoted Henry Beecher, saying that "there is the more reliable safeguard provided by the presence of an intelligent, informed, conscientious, compassionate, responsible investigator." ${ }^{15}$ Beecher believed in the significance of "a special relationship of trust between subject or patient and the investigator," stating, "the best approach concerns the character, wisdom, experience, honesty, imaginativeness and sense of responsibility of the investigator who in all cases of doubt or where serious consequences might remotely occur, will call in his peers and get the benefit of their counsel." This sentiment was echoed by Franz Ingelfinger: 16 "The subject's only real protection, the public as well as the medical profession must recognize, depends on the conscience and compassion of the investigator and his peers," and by Charles Weijer and his co-authors ${ }^{17}$ who thought that "the best way to monitor protocol adherence is a 
form of researcher self-regulation, with input from the IRB or from experts external to the institution."

We conclude this section by suggesting that more communication, not less as when one of the players does not share the information to which only he is privy, is the best protection for human subjects. Kenneth Goodman, ${ }^{18}$ focusing on IRBs' responsibility to minimize risks, thought that, for longer-term trials at least, IRB members should be educated about systematic science ${ }^{19}$ and its use in protecting subjects. He thought that IRBs "should have better means of communicating among themselves, investigators, sponsors, data safety monitoring boards, and others, as appropriate, especially on multi-center trials," because "what is clearer than ever is that high-quality review just at the end). Here, preliminary trends, not reaching usual - or conservatively adjusted - levels of significance may not dictate early termination of the trial, but may be of interest - and relevance - to both current participants and those considering participation. The general requirement quoted above applies to those already participating, but it seems clear that those considering participation are also entitled to upto-date information concerning the risks and possible benefits associated with the trial.

The regulations also clearly state that consent forms may require updating on the basis of preliminary observations. One needs not only promise to provide information that may impact on subjects' willingness to participate, but that information is to be provided

\section{While current or potential participants may not have a legal right to interim trial results, withholding such results may deprive them of valuable information - information that might well influence their decision to participate, or continue participation, in the trial.}

entails that subjects on trials will be informed of developments, new knowledge, and newly discovered risks that might affect their agreement to participate." The difficulty and importance of this task all but mandates an "all hands on deck" approach. A spirit of openness is evident when other conflicts of interest of PIs are managed. PIs are not forbidden to direct a study in which they may have conflicts of interest; they are expected to disclose the details of the conflict so that an effective management strategy can be developed. Why there should be special rules for managing conflicts involving data is not apparent.

\section{Apartheid Data Monitoring}

Our second concern is that information concerning early trends in the data is kept confidential by the DSMB. This information is not shared with sponsors, investigators, current and prospective trial participants, nor with those responsible for the clinical care of subjects. One of the "when appropriate" general requirements for informed consent (45 CFR 46.116(b) (5)) reads, "A statement that significant new findings developed during the course of the research which may relate to the subject's willingness to continue participation will be provided to the subject." One of the circumstances in which appropriateness seems especially transparent is an RCT in which outcome measures become available throughout the trial (and not formally, and in a timely manner. Mary Adams and Dennis Conrad ${ }^{20}$ quoted from an Office for Protection from Research Risks (OPRR) "Dear Colleague" letter regarding "substantive and meaningful" continuing review:

IRBs must evaluate the following: number of subjects accrued; a description of any adverse events or unanticipated problems involving risks to subjects or others; withdrawal of subjects from the research, or complaints about the research; a summary of any recent literature, findings, or other relevant information, especially information about risks associated with the research; and a copy of the current informed consent, containing accurate and complete information, including any significant new findings that may relate to the subject's willingness to continue participation.

We suppose that one might quibble about the meaning of "significant" here, but the word "statistically" is conspicuous by its absence, and the fact that this is a consent document seems to indicate that it be "significant to the subject." Less ambiguous are the words "accurate and complete." Complete has got to mean that nothing has been left out. In any case, it is difficult to see how IRBs are to discharge this duty if they are not informed of the new findings, irrespective of "sig- 
nificance." The Office for Human Research Protection (OHRP) recently confirmed this long-standing policy. From (very) recent OHRP Guidance, when reviewing the current informed consent document(s), the IRB should ensure that any significant new findings that may relate to the subject's willingness to continue participation are provided to the subject in accordance with HHS regulations at 45 CFR 46.116(b)(5). ${ }^{21}$

We have already reviewed Meinert's concerns about not allowing investigators to serve on DSMBs. In two other papers, he questioned the wisdom of masked monitoring, ${ }^{22}$ and confronted the problem of bias: ${ }^{23}$ "The risk of bias is nil (even if investigators see interim results) in masked trials where the masking is effective, and likely to be low (even in the absence of masking) with crisp treatment protocols and objective outcome measures." Here "masking" (or "blinding") refers to subjects or investigators or both not being aware of treatment assignments. We note that "objective outcome measures" should be the norm for studies that would even consider the use of a DSMB. The FDA guidelines are clear that studies involving symptom relief rarely warrant DSMBs; rather, more serious and objective - outcomes like death or cancer recurrence are typical.

Apartheid data monitoring has implications beyond the regulations and bias. Wells and colleagues ${ }^{24}$ made a not very subtle suggestion that DSMB secrecy might constitute fraudulent behavior, noting that fraud is defined as "an intentional misrepresentation, concealment or nondisclosure for the purpose of inducing another to part with some valuable thing...or to surrender a legal right." While current or potential participants may not have a legal right to interim trial results, withholding such results may deprive them of valuable information - information that might well influence their decision to participate, or continue participation, in the trial.

Wells and colleagues then present some examples showing that while interim results may not reach levels of significance calling for termination of the trial, these results would be compelling to "the reasonable man" trying to decide which of two treatments he prefers. Suppose that a clinical trial is well designed to compare two interventions, A and B. Assume that when $50 \%$ of the total number of patients have been seen, an interim analysis shows A is somewhat, but not significantly better than B with $\mathrm{p}=0.10$. Also suppose there are no safety concerns, i.e., the side-effect profiles of A and B are similar. The DSMB would have no reason not to allow the study to continue. However, an individual contemplating randomization might be keenly interested in the fact that, given $\mathrm{p}=0.10$ for $50 \%$ accrual, the relative risk of a patient getting infe- rior treatment is 8,450 times higher if the patient is treated with B..$^{25}$

An example structured somewhat differently, and involving much smaller numbers than would be expected with $50 \%$ accrual, was given by Richard Royall. ${ }^{26}$ Suppose in a trial of A vs. B, four patients were given $A$, and three of the four had a successful (S) outcome. B was also given to four patients, and no successes were observed. The p-value (using Fisher's exact test) is $\mathrm{p}=0.143$, which does not reach conventional (and surely not more conservative, "corrected") levels of significance. The trial would then continue, unchanged, the DSMB being the only ones aware of the results to date. However, making some reasonable assumptions about the probabilities of success for the two treatments and assuming that, initially, A and B are equally likely to be the better treatment (true equipoise), the data point strongly in the direction of treatment A. In particular, if the probabilities of success for $\mathrm{A}$ and $\mathrm{B}$ differ, and if $\mathrm{P}(\mathrm{S})$, the probability of a successful outcome, is 1.25 times higher for the more effective treatment, then, if we thought $\mathrm{P}(\mathrm{A}$ better $)=1 / 2$ before any data were collected, it is now true, given the data, that the odds that $\mathrm{A}$ is the better of the two treatments is 2:1. If we were considering participation in the trial, and knew that it was this likely that A were superior, we doubt that randomization would be an attractive option.

The lesson to be learned from these examples is that there is a difference between the amount of evidence required to attain statistical significance and that required to determine individual preference. As pointed out by Robert Veatch, ${ }^{27}$ "The standard used in rejecting a null hypothesis differs from the one used to make a forced choice between two treatment options." Rejection of a null hypothesis requires that the probability of doing so erroneously is controlled at a satisfactorily small level (often $5 \%$ ); less impressive differences suffice for deciding which of two treatments is currently preferred. A more elaborate discussion of the difference between "decisions" and "conclusions" was offered by John Tukey ${ }^{28}$ almost 50 years ago. This discussion deserves its own subsection.

\section{Decisions and Conclusions}

According to Tukey, decisions are associated with an "act for the present as if" attitude. One attempts to do as well as possible in a specific situation - to choose wisely among available gambles. Conclusions, on the other hand, are established with careful regard of evidence, but without regard to consequences of specific actions in specific circumstances. Conclusions are withheld until adequate evidence has accumulated. Subjects in trials face decisions: whether to participate 
or continue participation. They need to decide now, and they want to do as good as they can for themselves when making the choice. They are interested in current, accumulated data that are useful in helping them efits. Following this line of thought, Joseph Kadane ${ }^{30}$ suggested two principles to guide what a clinical trial should offer the subject: (1) whatever benefits the subject might reasonably be able to obtain outside the trial, and (2) share or use information developed during the course of the trial for the benefit of the subject. One way in which accumulating data might benefit participants would be to let them know about it, but there are other possibilities. Kadane did not share the results with subjects, but he used accumulating data to their benefit by restricting the treatment options that a particular subject might receive based on that subject's characteristics.

A more recent discourse on the decision/conclusion distinction was given by Deborah Hell-

decide - to choose wisely among the available gambles. Investigators, on the other hand, seeking conclusions, are less interested in the accumulating data; they are content to let it accumulate to the point where adequate evidence permits a conclusion to be drawn.

No one disputes the (societal) value of being able to reach a conclusion that would confidently guide clinical practice, but to insist that this be achieved by disadvantaging subjects is an affront to the Helsinkian admonition: "Concern for the interests of the subject must always prevail over the interests of science and society." As put by Veatch, "All would agree that it would be preferable to have more definitive data, but if a patient must be treated before such data become available, then both law and ethics require that the subject is told what the reasonable person would want to know, including the preliminary data trends." The examples presented by Wells et al. and Royall clearly show statistical significance need not be achieved before early results would influence decisions.

Veatch ${ }^{29}$ called this the "preliminary data problem," and concluded that studies in which preliminary data were not disclosed "would fail to satisfy the autonomy component of the respect for persons principle." This directs our attention to the Belmont troika: respect for autonomy would seem to require that the information presented to prospective participants be what they would consider to be useful to them, and up-todate. Concern for the rights and welfare of subjects as expressed in beneficence implies that participants not be disadvantaged as a result of their participation, and it is difficult to see how risks to the subject will be minimized and/or benefits maximized by not revealing what happened to earlier or concurrently enrolled subjects. Justice suggests that those accepting any risks associated with participation are entitled to share in the benefits of the research, and it is not clear that one necessarily need wait until the trial ends to reap ben- man. ${ }^{31}$ She started with the notion that, given a set of data, D, we can ask three questions:

(1) What does the evidence (D) show?

(2) Given this evidence, what should I believe?

(3) Given this evidence, what should I do?

Only the answer to the first question depends on the data alone. What one believes and what one does will depend on the data, but also on other factors: background information not present in the data. In any particular application, the answers to the three questions may appear at odds with one another. Suppose the data, the results of a diagnostic test, indicate that a disease is present. One might still believe that the disease is not present if the disease is extremely rare and the test has a relatively large false-positive rate. And, one might still reasonably choose to be treated for the disease if the disease is debilitating and the cost of treatment small. She thus argued that there is a distinction between criteria for justified belief and criteria for reasonable action; in particular, that the epistemic standard required to believe that $\mathrm{A}$ is better than B is much more difficult to meet than that to form a preference about the best treatment option for a particular patient. Participants (or potential participants) in a trial are concerned with what they should $d o$; trial investigators and sponsors want to know what to believe about the relative merits of the treatments. Less data are required to guide the decision than to firmly establish belief. Hellman put the argument as follows. Equipoise involves beliefs: one is in equipoise because one is unsure, given the evidence, what one should believe. For clinicians and for patients, however, the most important question is not, "What should I believe?" but "What should I do?" That there is uncertainty of belief does not mean that there is or should be uncertainty about how to act. 
Fred Gifford ${ }^{32}$ used slightly different terminology, but studied what he called the important "difference between (1) the amount of evidence that would be sufficient to warrant a preference for treatment $\mathrm{A}$ over treatment $\mathrm{B}$ for a given present patient right in front of you (the 'present patient' decision), and (2) the amount of evidence that would be sufficient to warrant the policy decision about how to treat not just this next patient but many future patients as well; that is, to stop the trial, announce the results, approve the drug, etc (the 'policy' decision)." He argued that the second decision (what we have been calling a "conclusion") required more evidence than the first since more was at stake. The consequences of being wrong would be of more moral impact, since it would affect greater numbers of people.

An even more recent discussion, again focusing on equipoise, distinguished between action-aims and outcome-aims. ${ }^{33}$ One has an action-aim insofar as one is disposed to do (now) what is best for her (present) patients; and an outcome-aim insofar as one is disposed to try (now) to bring about that she do (for the rest of her career) the best for her (present and future) patients. Winston Chiong argues that the existence of two different aims need not reflect two separate activities governed by different norms; rather they reflect a single activity in which the two aims constrain one another. Patient care is an activity encompassing both the care of current patients and those to be cared for in the future. Rather than advocating an absolutepriority view, he suggests a limited-priority position in which physicians give "somewhat greater weight" to the interests of current patients. This is based on a Kantian universalizability test in which one asks if we could reasonably want all physicians to act in a given way? If always, in all contexts, physicians gave absolute priority to their current patient, then medical education (students, while learning, are not at their "best") and clinical research (involving such things as placebos, blinding, and "unnecessary tests" to assess efficacy) would be impossible. A middle road seems preferable.

In the research context, the question, "What compromises in the care offered to subjects in a clinical trial can be justified by potential benefits to third parties?" is considered by asking what will it cost the current subject if the balance is shifted towards the third party. This is context-dependent and requires judgment. ${ }^{34}$ In our application, it is necessary to ascertain how much risks will be increased and/or autonomy diminished if interim results are kept secret. This will depend on the particulars of the trial.

\section{So, What's an IRB to Do?}

We suggest that how best to monitor RCTs will not have a single, unique, time-invariant answer. This follows from the fact that the problem is political in nature, and the political road moves in directions determined by continuing discussion, debate, and compromise. This was noted by Paul Meier, ${ }^{35}$ who, after detailing some of the statistical problems in monitoring, said, "It is clear that, although statistics has a role, the ethical problem of continuing or stopping an experiment is not primarily statistical. Neither is it a medical problem or a legal one. It is, in fact, a political problem... what sort of a society we wish to live in. How do we wish to deal with our fellows, and to be dealt with by them." The "right way to deal" will vary from trial to trial, from IRB to IRB, and even, for a given IRB and trial, there may be variability over time. We can therefore only offer some signposts along the way.

Robert Amdur ${ }^{36}$ suggested that IRBs confront the question of whether a DSMB should be employed right up front. He cites guidelines stating, "At the time of initial review, the IRB should determine whether a [DSMB] is required, and should also set a date for reevaluating the research." He points out that federal guidance does not establish criteria for determining when a DSMB should be used. He does, however, suggest, "Do not require a DSMB if it is reasonable to accept an alternative option.... The IRB should require a DSMB only in those situations where there is no other reasonable alternative." We concur. DSMBs should be employed only in rare circumstances and charged, first and foremost, with the protection of human subjects in accordance with ethical principles and to the satisfaction of IRBs.

There is no question that IRBs have a considerable say in these matters. The FDA guidance cited above stated that the IRB is entitled to know all it needs to know about a DSMB in order to do its job of protecting the rights and welfare of participants. To determine whether risks to subjects are minimized, "an IRB may appropriately request information about the approach to trial monitoring, including the basis for early termination....an IRB may appropriately inquire as to whether a DMC has been established and, if so, seek information about its scope and composition." Meinert added, "IRBs hold the trump cards, if they care to use them, when it comes to who monitors and how it is done." These "trump cards" give IRBs the ultimate say in that they need not - should not - approve projects with deficient plans for monitoring.

We continue with a discussion of what needs to be done if we are in one of the (rare) situations in which the IRB allows the use of a DSMB. Assuming that the use of a DSMB is appropriate in a given situation, we 
suggest that information concerning the existence and functioning of this body should be included in the consent form.

\section{Informed Consent}

Every IRB member worth her salt is able to mouth the anthem, "informed consent is a process." As noted by David Wendler and Jonathan Rackoff, ${ }^{37}$ "Many commentators have argued that informed consent should not be a single event at which subjects are informed and their consent obtained, but rather a process that continues for the duration of subjects' research participation." The basic idea is that, as time goes by, changes may occur in the nature of the research (risks, benefits, alternatives, even aims of the study), and/ or in subjects (medical status, interests, and preferences). If consent is truly a process, then, such changes of all potentially material information." Nevertheless, they argue that "an exception should be recognized to existing guidance on informed consent in clinical trials to explicitly permit withholding potentially material interim findings, subject to review by data monitoring committees, and to require disclosure of this practice to potential participants." Informed consent is seen to be critical to the possible ethical withholding of results as they accumulate. Tom Beauchamp and James Childress $^{39}$ put it this way: "As a rule, information about trends is not released prior to the completion or early termination of the RCT. This rule is justifiable if and only if prospective subjects understand and accept it as a condition for participating in the RCT."

However, despite the lip service paid to the process of informed consent, consent forms used in most studies employing DSMBs are less than forthcom-

\section{Where the use of a DSMB is either required, or can be justified in some other way, consent forms should be explicit about the existence and functions of the DSMB, including clear statements concerning the confidentiality of the accumulating data.}

would be communicated so that continuing participation remains informed. It may be argued that, at least in some cases, subjects might consent initially to continue participation in the face of certain specified possible changes. Pointing to possible alternative scenarios for the research process may enhance subjects' understanding of the research, but they should not be expected to assess exactly how they would react to hypothetical changes in the plan: instead, they should be allowed to change their minds. This follows directly from the Belmont principle of autonomy, and is one of the general requirements for informed consent ( 45 CFR 46.116(8)): "A statement that participation is voluntary, refusal to participate will involve no penalty or loss of benefits to which the subject is otherwise entitled, and the subject may discontinue participation at any time without penalty or loss of benefits to which the subject is otherwise entitled." This is federal law.

Even those who argue that, at least under certain conditions, it is ethical to keep interim findings of RCTs confidential, recognize that this is contrary to the regulations. Franklin Miller and David Wendler ${ }^{38}$ admit, "Confidential data monitoring withholds potentially material information from patients invited to enroll in RCTs and thus conflicts with current standards for informed consent which require disclosure ing about the way in which the process might work. Arthur Slutsky and James Lavery ${ }^{40}$ noted, "An explanation of the decision-making process of the DSMB and its potential implications for research subjects is currently not built into the informed-consent process in most trials." They propose that when a DSMB is involved,

prospective subjects should be informed about the board and told that any recommendation it makes to continue the trial, rather than stop it in the face of evidence suggestive of important differences in effectiveness among the treatments, might prolong exposure to a therapy that could turn out to be suboptimal or even harmful and could delay their access to an intervention that could ultimately prove to be more effective.

Stephen Cannistra ${ }^{41}$ added that consent forms should "explicitly state the criteria to be used for early study closure, thereby permitting the patient to make an informed decision regarding study participation."

Jerry Menikoff ${ }^{42}$ recently revisited these issues and found no improvement. He focused on "an example of what is clearly one of the best consent forms currently being used," one developed by a group of experts pur- 
posefully assembled by the National Cancer Institute (NCI) to study how consent forms should be written for cancer studies. The consent form, reproduced in its entirety on pages 105-112 of his book, contains the following statements concerning the DSMB (page110):

A Data Safety and Monitoring Board, an independent group of experts, will be reviewing the data from this research throughout the study. We will tell you about new information from this board or other studies that may affect your health, welfare, or willingness to stay on this study.

\section{Menikoff notes:}

Not only does this language not provide the warning that would make this practice ethical [a warning that new information will not be shared], but it appears to do just the opposite: to affirmatively commit the researchers to disclosing interim results that, if patients knew about them, might affect their willingness to be in the study.... Thus the consent form appears to actively deceive the subjects, incorrectly reassuring them of something (being told of interim results) that will in fact usually not occur....

He concludes:

Not giving them information that they would want under conditions where we acknowledge that there is a special duty to make sure consent is truly informed smacks of hypocrisy. In point of fact, failing to disclose information in the face of a clear duty to do so, with the intent to have a [sic] subjects enroll when they might otherwise not have done so, deserves an appropriate label: this is fraud, plain and simple.

While between the covers of Menikoff's book, we should follow his lead in pointing out that consent forms often do not accurately portray what is known (or strongly suspected) about experimental treatments; in particular, there is often a mismatch between what is stated in the study protocol (to be read by potential investigators and sponsors) and what is stated in the consent form (to be read by potential study participants). He gives an example in which the protocol stated that "pilot data...strongly suggest the possibility that some combination of chemotherapy and radiation therapy will have a significant impact on the surgical morbidity common encountered in managing head and neck neoplasms." In contrast, the consent form stated, "[S]urgery and/or radiation therapy are the standard treatments" and "other treatments are considered experimental." ${ }^{43}$ See also Veatch, who himself had a very hard time in obtaining a copy of the protocol for a study he was considering participation in. ${ }^{44}$

It seems clear, then, that the consent form should be more forthcoming about what the DSMB will and will not do about the results as they accumulate. Robert Levine ${ }^{45}$ indicated over 20 years ago how this might be done:

I suggest that we should go on to say [to someone considering enrolling in the study] that with time there will be increasing clarity that, e.g., A is superior to B. This superiority could manifest as a decreased probability of serious harms or an increased probability of benefits. However, for good reasons, we have designed the [study] so that neither the investigator nor the subject will be aware of this disparity until such time as the arbitrarily preselected criteria of significance are achieved. Thus, what I would ask the subject to consent to is an acceptance of the standards of proof agreed upon within the community of professionals. ${ }^{46}$

A somewhat less blunt statement of what effects the DSMB's actions might have is given by David Buchanan and Franklin Miller ${ }^{47}$ who suggest the wording:

This study is designed to determine whether patients with your condition who receive the study drug will have a reduced rate of new or recurrent experiences of the condition of at least 5 percent over a four-year period, compared to those who receive standard treatment. An independent Data and Safety Monitoring Board will review information concerning the effectiveness of the study drug at regular intervals during the study. The DSMB will stop the study immediately if evidence indicates that subjects in either study group have a higher rate of death [or "stop the study if there is firm evidence that the reduction in disease occurrence is greater than 5 percent" or "stop the study if there is an unacceptably large difference in rates of the targeted condition.”]

\section{Summary and Conclusions}

The main thrust of this paper has been to call into question the unchecked growth of the "DSMB industry." While recognizing that DSMBs may offer certain scientific advantages in some cases, we have argued that the small (scientific) gains that might be realized are accompanied by unacceptable (ethical) costs. As noted by Beauchamp and Childress, the ethical problems inherent in the practice of withholding informa- 
tion from participants do not "disappear" when one uses a DSMB. They state,

This differentiation of roles by using a monitoring committee, particularly in a double-blind RCT, is procedurally sound, but it relocates rather than resolves some central ethical questions. The committee must still determine when, if ever, it is legitimate to impose risks on current patients in order to benefit future ones by establishing with a high degree of certainty the superiority of one treatment over another or over a placebo. A committee will likely take the perspective that clinical equipoise must have been eliminated from the perspective of the expert medical community. However, the individual physician and his or her patient may be concerned primarily with whether clinical uncertainty has been eliminated for them.

We call for decelerated use of DSMBs and a careful re-examination of the issues raised in this paper so that we may be able to develop more "participantfriendly" guidelines for how best to use accumulating data in clinical trials. In the short term, however, we recognize that DSMBs will not completely disappear, their use even being required in certain situations. We would hope that in those situations where the use of a DSMB is either required, or can be justified in some other way, consent forms should be explicit about the existence and functions of the DSMB, including clear statements concerning the confidentiality of the accumulating data.

Finally, we have pointed to the pivotal role played by the principal investigator in clinical trials. No one knows more about the trial than the primary investigator (PI), who is uniquely positioned to interpret the data he or she is collecting for the purposes he or she has set forth. To usurp the power of the PI to evaluate the data as they accumulate is to "shoot oneself in the foot," and, importantly, will serve to further alienate PIs from the "regulatory police."

DSMBs do not write (or update) consent forms; investigators do. Nor do DSMBs approve consent forms; IRBs do. It is difficult to see how we can expect consent forms to be accurate, complete, and up-todate when the PI and IRB are deprived of the information needed to produce them. Blaming the PI for not producing the best consent form possible (and we believe potential subjects deserve this) under these conditions violates the oft-heard ethical maxim "ought implies can." The consent process is widely recognized to be the cornerstone of ethical research. If potential subjects do not trust the PI to be entirely forthcoming during this process, then we risk alienating society from the research process itself, an outcome that can only be described as "lose-lose."

\section{References}

1. These are also known as Data Safety Committees (DSCs), Independent Data Monitoring Committees (IDMCs), Data Monitoring Committees (DMCs) and Data Review Boards (DRBs).

2. P. Tharmanathan, M. Calvert, J. Hampton, and N. Freemantle, "The Use of Interim Data and Data Monitoring Committee Recommendations in Randomized Controlled Trial Reports: Frequency, Implications and Potential Sources of Bias," BMC Medical Research Methodology 8 (2008), available at <http:// www.biomedcentral.com/1471-2288/8/12> (last visited July 20, 2009).

3. S. S. Ellenberg, T. R. Fleming, and D. L. DeMets, Data Monitoring Committees in Clinical Trials (New York: Wiley, 2003).

4. S. J. Green, T. R. Fleming, and J. R. O'Fallon, "Policies for Study Monitoring and Interim Reporting of Results," Journal of Clinical Oncology 5, no. 9 (1987): 1477-1484.

5. R. J. Wells, P. S. Gartside, and C. L. McHenry, "Ethical Issues Arising When Interim Data in Clinical Trials Is Restricted to Independent Data Monitoring Committees," IRB: A Revierw of Human Subjects Research 22, no. 1 (2000): 7-11.

6. S. J. L. Edwards, R. J. Lilford, and J. Hewison, "The Ethics of Randomized Controlled Trials from the Perspectives of Patients, the Public and Healthcare Professionals," BMJ 317, no. 4 (1998): 1209-1212.

7. See Ellenberg et al., supra note 3. One reason for this is that the NIH requires that a DSMB monitor every phase III trial.

8. Food and Drug Administration, Guidance for Clinical Trial Sponsors: Establishment and Operation of Clinical Trial Data Monitoring Committees, Rockville, MD, 2005.

9. See Ellenberg et al., supra note 3.

10. C. L. Meinert, "Clinical Trials and Treatment Effects Monitoring," (with discussion), Controlled Clinical Trials 19 (1998): 515-543.

11. B. Freedman, "Equipoise and the ethics of clinical research," New England Journal of Medicine 317, no. 3 (1987): 141-145.

12. S. Hellman and D. S. Hellman, "Of Mice, but Not Men: Problems of the Randomized Clinical Trial," New England Journal of Medicine 324, no. 22 (1991): 1589-1592.

13. R. J. Lilford, D. Braunholtz, S. S. Edwards, and A. Stevens, "Monitoring Clinical Trials - Interim Data Should Be Publicly Available," BMJ 323, no. 7310 (2001): 441-442.

14. They cite the ISIS 2 example, where initially skeptical physicians moved into equipoise.

15. Both the NBAC and Beecher are quoted in R. Michels, "Are Research Ethics Bad for Our Mental Health?” Nerw England Journal of Medicine 340, no. 18 (1999):1427-1430.

16. F. J. Ingelfinger, "Informed (but Uneducated) Consent," New England Journal of Medicine 287, no. 6 (1972): 465-466.

17. C. Weijer, S. H. Shapiro, A. Fuks, K. C. Glass, and M. Skrutkowska, "Monitoring Clinical Research: An Obligation Unfulfilled," Canadian Medical Association Journal 152, no. 12 (1995): 1973-1979.

18. K. W. Goodman, Ethics and Evidence-Based Medicine (Cambridge: Cambridge University Press, 2003).

19. By this is meant systematic reviews of the literature, aka metaanalysis.

20. M. S. Adams and D. A. Conrad, "Annual Review: Observed Deficiencies and Suggested Corrections," IRB: A Review of Human Subjects Research 18, no. 5 (1996): 1-6.

21. Available at <www.hhs.gov/ohrp/humansubjects/guidance/ contrev0107.htm > (last visited July 7, 2009).

22. C. L. Meinert, "Masked Monitoring in Clinical Trials - Blind Stupidity?” Ner England Journal of Medicine 338, no. 16 (1998): 1381-1382.

23. C. L. Meinert, "IRBs and Randomized Clinical Trials," IRB: $A$ Revier of Human Subjects Research 20, no. 1 (1998): 9-12. 
24. See Wells et al., supra note 5 .

25. To be fair, this is based on the p-values 0.169 that by study's end A would be favored, and 0.00002 that B would be favored. The chance that neither would be favored is 0.8309 .

26. R. M. Royall, "Ethics and Statistics in Randomized Clinical Trials," (with discussion), Statistical Science 6, no. 1 (1991): 52-88.

27. R. M. Veatch, "Comment," Controlled Clinical Trials 19, no. 6 (1998): 532-533.

28. J. Tukey, "Conclusions vs. Decisions," Technometrics 2, no. 4 (1960): 423-433.

29. R. M. Veatch, "Longitudinal Studies, Sequential Design, and Grant Renewals: What to Do with Preliminary Data,"IRB: $A$ Review of Human Subjects Research 1, no. 3 (1979): 1-3.

30. J. B. Kadane, ed., Bayesian Methods and Ethics in a Clinical Trial Design (New York: Wiley, 1996).

31. D. Hellman, "Evidence, Belief and Action: The Failure of Equipoise to Resolve the Ethical Tension in the Randomized Clinical Trial," Journal of Law, Medicine छ Ethics 30, no. 2 (2002): 375-380.

32. F. Gifford, "Freedman's 'Clinical Equipoise' and 'Sliding-Scale All-Dimensions-Considered Equipoise," Journal of Medicine Eं Philosophy 25, no. 4 (2000): 399-426.

33. W. Chiong, "The Real Problem with Equipoise," American Journal of Bioethics 6, no. 4 (2006): 37-47.

34. Chiong suggested a rough-and-ready test for the universalizability of some practice: whether we would be willing to imagine ourselves, our loved ones, or our peers on the receiving end of some treatment. What would it be like if everybody did that?

35. P. Meier, "Statistics and Medical Experimentation," Biometrics 31, no. 2 (1975): 511-529.

36. R. J. Amdur, "Provisions for Data Monitoring," in R. J. Amdur and E. A. Bankert, eds., Institutional Review Board: Management and Function (Sudbury, MA: Jones and Bartlett, 2002): 191-196.

37. D. Wendler and J. Rackoff, "Consent for Continuing Research Participation: What Is It and When Should It Be Obtained?" IRB: Ethics \& Human Research 24, no. 2 (2002): 1-6.
38. F. G. Miller and D. Wendler, "Is It Ethical to Keep Interim Findings of Randomized Controlled Trials Confidential?" Journal of Medical Ethics 34, no. 4 (2008): 198-201.

39. T. L. Beauchamp and J. F. Childress, Principles of Biomedical Ethics, 5th ed. (New York: Oxford University Press, 2001).

40. A. S. Slutsky and J. V. Lavery, "Data Safety and Monitoring Boards," New England Journal of Medicine 350, no. 11 (2004): 1143-1147.

41. S. A. Cannistra, "The Ethics of Early Stopping Rules: Who Is Protecting Whom?” Journal of Clinical Oncology 22, no. 9 (2004): 1542-1545.

42. J. Menikoff, What the Doctor Didn't Say: The Hidden Truth about Medical Research (New York: Oxford University Press, 2006).

43. The consent form also failed to mention that that the patient could get the chemotherapy from a doctor, outside of the study, and thus be sure of getting this, instead of the $50 \%$ chance in the study. See $i d$., at 130 .

44. R. M. Veatch, "The Right of Subjects to See the Protocol," IRB: Ethics छீ Human Research 24, no. 4 (2002): 6-8.

45. R. J. Levine, Ethics and Regulation of Clinical Research, 2nd ed. (New Haven: Yale University Press, 1986).

46. This differs from the "reasonable person" standard, as described by Menikoff (supra note 42, at 92), who suggests that "...the appropriate standard for determining disclosure in the research setting should always be the reasonable person standard." Beauchamp and Childress (supra note 2, at 327) thought DSMBs should "supply physicians and patients with significant safety and therapeutic information that is relevant to a reasonable person's decision to remain in or withdraw from the trial.” Reasonable people need not insist on statistical significance before they are willing to act.

47. D. Buchanan and F. G. Miller, "Principles of Early Stopping of Randomized Trials for Efficacy: A Critique of Equipoise and an Alternative Nonexploitation Ethical Framework," Kennedy Institute of Ethics Journal 2, no. 2 (2005): 161-178. 